\title{
La modellazione microstrutturale di materiali a struttura eterogenea: princìpi ed applicazioni
}

\author{
Luca Collini \\ Università di Parma, Dipartimento di Ingegneria Industriale, v.le G.P. Usberti 181/A, 43100 Parma \\ luca.collini@unipr.it
}

Riassunto. Molti problemi della Meccanica e Fisica dei Solidi e della Scienza dei Materiali, non sono facilmente risolvibili con gli approcci tradizionali. Oltre allo studio delle proprietà effettive dei solidi eterogenei, vi è la crescente necessità di incorporare un maggiore numero di informazioni sui meccanismi di deformazione e danneggiamento generati alla microscala, anche per i materiali abitualmente considerati omogenei. Micromeccanismi di cavitazione e concentrazioni locali di tensione e deformazione, sono indispensabili per spiegare fenomeni non-lineari come la rottura di fatica o il cedimento duttile, altrimenti non inquadrabili con approcci classici di tensioni e deformazioni medie. La micromeccanica si occupa della determinazione precisa, o di una stima accurata, di grandezze di campo microstrutturali locali. In questo lavoro sono illustrati i princìpi che sono alla base dell'approccio micromeccanico, come i concetti di multiscala, di distribuzione statistica delle fasi, di descrizione mediante volumi di riferimento e di omogeneizzazione e localizzazione, e, attraverso alcune applicazioni pratiche delle principali tecniche di modellazione, sono illustrati e discussi criticamente i risultati della ricerca effettuata su varie strutture di ghisa nodulare.

ABSTRACT. There are many problems in Solid Mechanics, Physics of Solids and Materials Science that can not been solved using conventional approaches. Besides the effective properties of non-homogeneous bodies, there is an increasing need for incorporating more physical information about small-scale mechanisms of deformation and damage into phenomenological models of plasticity and rupture of materials which are usually considered homogeneous. Cavitation, local stress or strain concentrations at small scale are essential to explain nonlinear phenomena, such as fatigue or ductile rupture, which are not understandable from the simple point of view of average stresses or strains. Precise determination or accurate estimation of local fields is the domain of micromechanics. This work treats the basic concepts of the micromechanics in a simple way, with some practical suggestion on the most commonly used modelling techniques and some applications made by the author on various nodular cast iron microstructures.

KEYwORDs. Modellazione micromeccanica; Materiali a struttura eterogenea; Compositi; Comportamento elasto-plastico.

\section{INTRODUZIONE ALLA MECCANICA DEI MATERIALI MICROSTRUTTURATI}

\section{Materiali microstrutturati e concetto di scala di osservazione}

T a Micro- e Meso-Meccanica del Continuo è una branca relativamente giovane della Meccanica dei Materiali, che studia il comportamento meccanico dei materiali a livello microstrutturale. Molti dei materiali di interesse industriale ed ingegneristico, e la maggior parte dei materiali "naturali", presentano una struttura eterogenea, formata cioè da più costituenti o "fasi", distinguibili ad una certa (ridotta) scala di osservazione. In altre parole, ciò che al 
nostro occhio può sembrare un solido continuo ed omogeneo, se analizzato ad un'altra lunghezza di scala si rivela formato da più costituenti continui, che a loro volta possono essere eterogenei (per proprietà, orientamento, ecc.) aumentando ulteriormente la definizione della scala di osservazione. È questo un concetto fondamentale nello studio della meccanica dei materiali a livello microscopico. Tipici esempi di materiali eterogenei sono i materiali compositi, fibro-rinforzati o particellari, i materiali porosi, gli stessi metalli policristallini, ma anche il legno e l'osso.

Una diretta conseguenza di questo concetto è l'approccio cosiddetto multiscala allo studio del comportamento meccanico dei materiali microstrutturati, associato a fenomeni e meccanismi peculiari della dimensione in cui si conduce l'analisi [1, 2].

Un gran numero di problemi della Meccanica dei Solidi e della Scienza dei Materiali non può di fatto essere risolto tramite gli approcci convenzionali, poiché alla base delle effettive proprietà meccaniche di un materiale eterogeneo vi è la crescente necessità di incorporare informazioni fisiche sui micromeccanismi di deformazione e danneggiamento all'interno di modelli fenomenologici di plasticità o rottura, caratteristici dei materiali omogenei. I concetti di nucleazione di vuoti o di localizzazione delle deformazioni a livello microscopico sono alla base di fenomeni non-lineari come la fatica nei materiali, o la rottura duttile, che non sono spiegabili dal solo punto di vista delle tensioni e delle deformazioni medie o mediate. La precisa determinazione delle grandezze di campo locali nel materiale è l'ambito di studio della micromeccanica.

I processi di deformazione elasto-plastica sono convenzionalmente trattati facendo riferimento alla Meccanica del Continuo, che si basa su di una descrizione macroscopica o effettiva del comportamento meccanico del materiale. Tale approccio, in cui rientra anche la stessa teoria delle dislocazioni, mostra dei limiti nell'interpretare i meccanismi plastici in un materiale a microstruttura eterogenea. Per fare ciò l'analisi deve affinarsi in definizione considerando fattori aggiuntivi a livello microstrutturale, come il comportamento di un singolo elemento del materiale (cristallo, grano, fase...), la mutua interazione tra questi, il comportamento delle zone di confine, la formazione di nuove strutture interne durante la deformazione (ad es. celle, bande di scorrimento, blocchi o altre sottostrutture).

Da quanto detto appare evidente l'importanza che riveste la dimensione, o scala, alla quale si studia il comportamento di un materiale. La micromeccanica definisce quindi una gerarchia di livelli dimensionali organizzati, appartenenti a diversi ordini di grandezza [3, 4]. Ad ogni livello, o scala, si distingue una determinata organizzazione delle fasi. In Fig. 1 è schematizzato questo concetto, con diretto riferimento alla struttura tipica di una ghisa nodulare a matrice mista ferriticoperlitica.

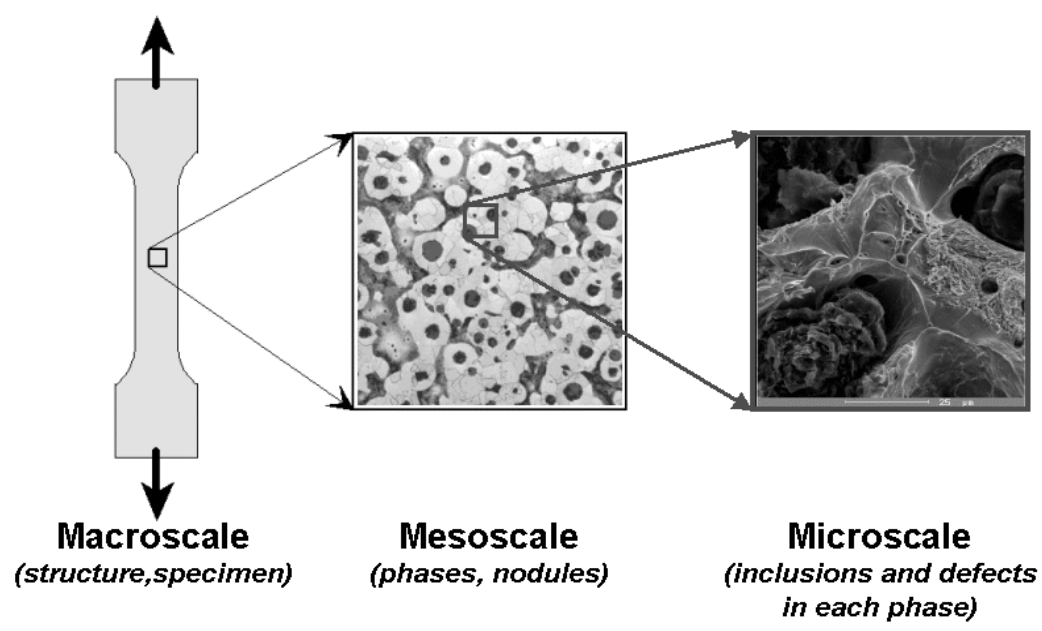

Figura 1: Scale di osservazione associate alla struttura di una ghisa nodulare.

La macroscala, Fig. 1 a sinistra, identifica il comportamento meccanico macroscopico del materiale, ad esempio la sua resistenza determinata con una prova di trazione, di tenacità a frattura, etc. La fenomenologia associata alla macroscala definisce le proprietà solitamente impiegate nella progettazione meccanica. Al livello della microscala, Fig. 1 a destra, gli elementi caratteristici sono invece inclusioni, porosità, disposizioni irregolari o difetti della struttura (vacanze, dislocazioni). Nel caso illustrato relativo alla ghisa nodulare, alla microscala si manifesta ad esempio il meccanismo di danneggiamento duttile (cavitazione plastica e successiva instabilità della matrice attorno ai noduli di grafite, tipico delle ghise ferritiche): la microscala evidenzia quindi le cause e i meccanismi alla base del comportamento del materiale. Una scala intermedia alle precedenti è la mesoscala. Fig. 1 al centro mostra una tipica sezione metallografica della ghisa sferoidale. La mesoscala rappresenta il livello in cui s’individuano le fasi presenti o gli elementi caratteristici della microstruttura. La 
mesoscala possiede una sua peculiarità: essa ammette la descrizione del comportamento di ogni singola fase come un continuo. Nella ghisa ad esempio è individuata la fase ferritica (regioni di colore chiaro), quella perlitica (regioni grigio scuro) e la grafite che durante il raffreddamento della massa fusa precipita sotto forma di noduli o sferoidi. In funzione della percentuale delle due fasi - ferrite e perlite - presenti nella matrice, le zone ferritiche sono disposte o attorno ad un singolo nodulo di grafite (configurazione bull's eye), o in aree più estese racchiudono un numero maggiore di sferoidi. La mesoscala identifica quindi un altro aspetto essenziale del materiale: la disposizione delle fasi.

L'analisi del comportamento meccanico di materiali multi-fase o a microstruttura eterogenea su una determinata scala, richiede in genere strumenti di modellazione. I modelli sono volti alla comprensione dei micro-meccanismi che governano il comportamento del solido, manifestato su scala macroscopica. Essi forniscono indicazioni sul comportamento d'insieme di un solido disomogeneo, a partire dalla conoscenza del comportamento delle singole fasi e della loro interazione, [5].

La microstruttura della maggior parte dei materiali eterogenei di interesse ingegneristico è solitamente troppo complessa, dal punto di vista puramente geometrico, per essere descritta nel dettaglio in modo deterministico. La disposizione delle fasi deve necessariamente essere trattata in modo statistico, e descritta mediante appropriate funzioni di distribuzione e correlazione. La struttura eterogenea di un materiale può infatti essere considerata "statisticamente omogenea", e la descrizione statistica dell'arrangiamento non dipende dalla posizione in cui esso è valutato. Questo è un altro concetto di fondamentale importanza alla base dell'approccio micromeccanico.

Per questi sistemi statisticamente omogenei, è lecito definire delle proprietà medie sul volume, indipendenti dalla grandezza e dalla posizione del volume considerato, ammesso che esso sia "abbastanza grande". Un tale volume che contiene tutte le informazioni necessarie e sufficienti per la descrizione statistica di una data microstruttura è detto volume di riferimento, o Reference Volume Element (RVE). Una microstruttura statisticamente omogenea può essere isotropa se i descrittori statistici sono degli invarianti rotazionali, o statisticamente anisotropa se vi sono direzioni preferenziali nella geometria, orientazione o posizione dei costituenti, [6].

\section{Omogeneizzazione e localizagione.}

Una pratica comune nel trattare i materiali compositi è quella di sostituire idealmente il comportamento della loro struttura disomogenea con quello di un materiale omogeneo equivalente o, per meglio dire, omogeneizzato. La maggior parte delle proprietà macroscopiche può essere determinata sperimentalmente, ma alcune di esse, in pratica, non sono determinabili o sono molto difficili da misurare. Un'intera area della Meccanica dei Solidi si è occupata, negli ultimi 30 anni, della teoria dei compositi e della possibilità di predire le loro proprietà effettive a partire dal comportamento di ciascun costituente, o fase, e dalla loro disposizione, o microstruttura. Dai primi lavori di Hill [7] e Hashin e Shtrikman [8], notevoli progressi si sono avuti nello studio del comportamento lineare ed elastico dei materiali, mentre molto meno è stato proposto per quanto riguarda i fenomeni non lineari, come la plasticità e il creep.

Analizziamo il caso più semplice di materiale microstrutturato a cui sono associate due scale caratteristiche, la macroscala e la scala, molto più piccola, a cui il materiale è eterogeneo, che abbiamo chiamato microscala.

Alla macroscala il comportamento del materiale è descritto in maniera convenzionale, cioè come quello di un equivalente continuo omogeneo opportunamente scelto. Sforzi e deformazioni hanno dunque andamenti continui, che dipendono dalle variazioni di geometria e dalle condizioni di carico. In assenza di macroscopiche variazioni del gradiente di queste grandezze (si parla di slow variations), nessuna variazione è evidente alla microscala, dove l'eterogeneità del materiale produce microscopiche, localizzate fluttuazioni (fast variations). I tensori di tensione e deformazione, $\boldsymbol{\sigma}$ e $\boldsymbol{\varepsilon}$, funzione del vettore di posizione $\mathbf{x}$, possono essere allora pensati come la somma di grandezze di campo slow $(\langle\sigma\rangle,\langle\varepsilon\rangle)$ e fast $\left(\sigma^{\prime}, \varepsilon^{\prime}\right)$ :

$$
\begin{aligned}
& \sigma(\mathbf{x})=\langle\sigma\rangle+\sigma^{\prime}(\mathbf{x}) \\
& \varepsilon(\mathbf{x})=\langle\varepsilon\rangle+\varepsilon^{\prime}(\mathbf{x})
\end{aligned}
$$

Nel caso, peraltro comune, di materiali eterogenei che mostrano una distinzione sufficientemente definita tra le lunghezze di scala, è possibile scrivere la relazione:

$$
\int_{\Omega_{S}} \sigma^{*}(\mathbf{x}) \varepsilon^{*}(\mathbf{x}) d \Omega=\left\langle\sigma^{*} \varepsilon^{*}\right\rangle=\left\langle\sigma^{*}\right\rangle\left\langle\varepsilon^{*}\right\rangle
$$

valida per ogni campo di tensione statisticamente valido $\sigma^{*}$ e campo di deformazione cinematicamente ammissibile $\varepsilon^{*}$, [7]. Nell'Eq. (2), il simbolo \langle\rangle indica che una quantità è mediata sul volume di riferimento, e $\Omega_{\mathrm{S}}$ è il volume di controllo, grande almeno quanto il RVE. L'Eq.(2) è nota come la condizione di macro-omogeneità di Hill o di Hill-Mandel. Essa implica sostanzialmente che la densità media di energia di deformazione sul volume di un solido elastico disomogeneo, 
possa essere ottenuta da tensioni e deformazioni mediate sul volume, ammesso che le macro- e microscale siano sufficientemente diverse. Ciò è molto importante, poiché rende possibile il concetto di materiale omogeneo equivalente dal punto di vista energetico al corrispettivo materiale microstrutturato.

Per ciascuna regione di un materiale disomogeneo, i campi di tensione e deformazione locali $\boldsymbol{\sigma}(\mathbf{x})$ e $\boldsymbol{\varepsilon}(\mathbf{x})$ e le corrispondenti risposte macroscopiche $\langle\sigma\rangle$ e $\langle\varepsilon\rangle$, possono essere formalmente messi in relazione mediante le espressioni di localizzazione (o proiezione):

$$
\begin{aligned}
& \varepsilon(\mathbf{x})=\mathbf{A}(\mathbf{x})\langle\varepsilon\rangle \\
& \sigma(\mathbf{x})=\mathbf{B}(\mathbf{x})\langle\sigma\rangle
\end{aligned}
$$

Se la regione si presenta sufficientemente estesa e non contiene significativi gradienti nelle distribuzioni di tensione e deformazione macroscopiche, le relazioni di omogeneizzazione possono scriversi nella forma:

$$
\begin{aligned}
& \langle\varepsilon\rangle=\frac{1}{\Omega_{s}} \int_{\Omega_{s}} \varepsilon(\mathbf{x}) d \Omega=\frac{1}{2 \Omega_{s}} \int_{\Gamma_{s}}\left[\mathbf{u}(\mathbf{x}) \otimes \mathbf{n}_{\Gamma}(\mathbf{x})+\mathbf{u}(\mathbf{x}) \otimes \mathbf{n}_{\Gamma}(\mathbf{x})\right] d \Gamma \\
& \langle\sigma\rangle=\frac{1}{\Omega_{s}} \int_{\Omega_{s}} \sigma(\mathbf{x}) d \Omega=\frac{1}{\Omega_{s}} \int_{\Gamma_{s}}[\mathbf{t}(\mathbf{x}) \otimes \mathbf{x}] d \Gamma
\end{aligned}
$$

dove $\Gamma_{\mathrm{s}}$ è la superficie della regione in esame, $\mathbf{n}_{\Gamma}$ il vettore normale alla superficie, $\mathbf{u}$ il vettore di spostamento, $\mathbf{t}(\mathbf{x})=\sigma(\mathbf{x}) \mathbf{n}_{\Gamma}$ il vettore di trazione sulla superficie, e $\otimes$ il prodotto diadico tra vettori o diade (se $\mathbf{a}=a_{\mathrm{x}} \mathbf{i}+\mathrm{a}_{\mathrm{y}} \mathbf{j}+\mathrm{a}_{\mathrm{z}} \mathbf{k}$ e $\mathbf{b}=\mathrm{b}_{\mathrm{x}} \mathbf{i}+\mathrm{b}_{\mathrm{y}} \mathbf{j}+$ $\left.b_{z} \mathbf{k}, \mathbf{a} \otimes \mathbf{b}=a_{x} b_{x} \mathbf{i i}+a_{x} b_{y} \mathbf{i j}+a_{x} b_{z} \mathbf{i} \mathbf{k}+a_{y} b_{x} \mathbf{j} \mathbf{i}+a_{y} b_{y} \mathbf{j} \mathbf{j}+a_{y} b_{z} \mathbf{j} \mathbf{k}+a_{z} b_{x} \mathbf{k i}+a_{z} b_{y} \mathbf{k j}+a_{z} b_{z} \mathbf{k} \mathbf{k}\right)$. I tensori $\mathbf{A}(\mathbf{x})$ e $\mathbf{B}(\mathbf{x})$ sono detti tensori di concentrazione della tensione e della deformazione (o funzioni di influenza). Si noti che i campi di tensione e deformazione medie sono univocamente determinati dai campi di spostamento e di trazione, dato che i costituenti sono omogenei e non presentano difetti o discontinuità (come fessurazioni) al loro interno.

È importante notare che le equazioni (1) e (4) implicano che per un volume di integrazione abbastanza grande, le medie delle fluttuazioni sul volume si annullino:

$$
\frac{1}{\Omega_{S}} \int_{\Omega_{S}} \varepsilon^{\prime}(\mathbf{x}) d \Omega=\frac{1}{\Omega_{S}} \int_{\Omega_{S}} \sigma^{\prime}(\mathbf{x}) d \Omega=0 .
$$

Importanti applicazioni delle analisi di omogeneizzazione sono la caratterizzazione di materiali, come ad esempio la simulazione di semplici prove meccaniche di carico (diagramma tensione-deformazione), e modelli costitutivi micromeccanici in grado di riprodurre il comportamento del materiale comunque caricato. Data la generale complessità e la casualità delle microstrutture reali, delle semplificazioni ed approssimazioni vengono solitamente impiegate nei processi di omogeneizzazione e localizzazione. Le reali espressioni per $\mathbf{A}(\mathbf{x}), \mathbf{B}(\mathbf{x}), \boldsymbol{\varepsilon}(\mathbf{x}), \sigma(\mathbf{x})$, etc. non sono di solito determinabili. Tipicamente, tali approssimazioni sono basate sull'ipotesi di ergodicità degli stati di tensione e deformazione (un processo si dice ergodico quando, per un tempo che tende ad infinito, passa in ogni suo stato una percentuale di tempo pari alla probabilità di trovarsi in quello stato, cioè, in altre parole, è statisticamente omogeneo). Quindi si considera che volumi di materiale abbastanza estesi, scelti in maniera casuale, rispondano con lo stesso comportamento medio del materiale continuo che, di rimando, rappresenta le proprietà globali o effettive della struttura eterogenea.

Idealmente, il volume di omogeneizzazione dovrebbe essere scelto come volume di riferimento, ovvero un sotto-volume di $\Omega_{\mathrm{S}}$, che è statisticamente rappresentativo della microgeometria del materiale. Questo elemento di volume deve essere abbastanza esteso per permettere un campionamento significativo delle grandezze di campo di interesse, ma anche sufficientemente piccolo affinché risultino trascurabili i gradienti macroscopici. Nella pratica, può risultare difficoltoso studiare, e, di fatto, anche identificare, un RVE adatto a descrivere fedelmente la microstruttura del materiale eterogeneo; devono quindi essere prese in considerazione approssimazioni e ipotesi semplificative, come verrà di seguito mostrato in alcune applicazioni pratiche di modellazione microstrutturale.

\section{APPROCCI E METODI DELLA MODELLAZIONE MICROMECCANICA}

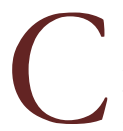

ome descritto nella precedente sezione, le tecniche di omogeneizzazione sono volte a determinare la risposta di un piccolo volume rappresentativo di un materiale a struttura eterogenea sotto l'azione di prescritte condizioni meccaniche o termiche, deducendo da esso le proprietà effettive del materiale. L'applicazione più tipica di questo 
tipo di analisi è la caratterizzazione del materiale, ciò̀ la simulazione della sua risposta complessiva se sottoposto ad un semplice carico, come ad esempio la prova di trazione uniassiale.

Molte procedure di omogeneizzazione possono essere impiegate anche direttamente sotto forma di modelli costitutivi del materiale, basati sulla microstruttura e atti a descrivere il macro-comportamento mettendo in relazione i tensori di tensione e deformazione completamente omogeneizzati. Confrontati con leggi costitutive semi-empiriche, tali modelli micromeccanici possiedono sia una chiara base fisica, sia l'intrinseca capacità di "zoomare" le quantità localizzate in ciascuna fase adottando le procedure di localizzazione. In questo modo è possibile determinare ad esempio il flusso plastico o il danno locali, quando la risposta macroscopica o lo stato di un campione o di una struttura sono noti a priori. Oltre alla caratterizzazione di un materiale o alla realizzazione di modelli costitutivi, la micromeccanica del continuo vede altre importanti applicazioni; tra tutte lo studio di fenomeni locali in materiali eterogenei, come l'evoluzione del danno su scala microscopica, l'innesco e la crescita di cricche, gli stati di tensione tra le interfacce macroscopiche e le superfici libere, gli effetti di instabilità locali, l’interazione tra cambiamenti di fase e microtensionamenti.

Analisi di questo tipo possono venire condotte con diverse tecniche e procedure pratiche; in questa sezione si darà una descrizione di alcune di esse, soffermandosi maggiormente su quelle impiegate dall'autore.

È conveniente suddividere le tecniche di modellazione microstrutturale in due grandi categorie. La prima di esse raggruppa i metodi che descrivono microgeometrie di materiali eterogenei sulla base di (limitate) informazioni statistiche, la seconda categoria abbraccia le metodiche di modellazione basate su microstrutture reali discrete.

\section{Metodi statistici}

Fanno parte di questa categoria gli approcci di tipo Mean Field e i metodi variazionali.

Negli approcci di tipo Mean Field (MFAs) le grandezze di campo sono approssimate con le loro medie $\langle\sigma\rangle^{(p)},\langle\varepsilon\rangle^{(p)}$ per ciascuna fase $p$ : si parla in questo caso di piecewise (phasewise) uniform stress and strain fields; le relazioni di localizzazione assumono la forma:

$$
\begin{aligned}
& \langle\varepsilon\rangle^{(p)}=\overline{\mathbf{A}}^{(p)}\langle\varepsilon\rangle \\
& \langle\sigma\rangle^{(p)}=\overline{\mathbf{B}}^{(p)}\langle\sigma\rangle
\end{aligned}
$$

e quelle di omogeneizzazione:

$$
\begin{array}{llll}
\langle\varepsilon\rangle^{(p)} & =\frac{1}{\Omega^{(p)}} \int_{\Omega^{(p)}} \varepsilon(\mathbf{x}) d \Omega & \text { con } & \langle\varepsilon\rangle=\sum_{p} V^{(p)}\langle\varepsilon\rangle^{(p)} \\
\langle\sigma\rangle^{(p)}=\frac{1}{\Omega^{(p)}} \int_{\Omega^{(p)}} \sigma(\mathbf{x}) d \Omega & \text { con } & \langle\sigma\rangle=\sum_{p} V^{(p)}\langle\sigma\rangle^{(p)}
\end{array}
$$

dove l'indice ( $\mathrm{p})$ indica una data fase del materiale, $\Omega(\mathrm{p})$ il suo volume, e $\mathrm{V}^{(\mathrm{p})}=\Omega(\mathrm{p}) / \Sigma_{\mathrm{k}} \Omega_{(\mathrm{k})}$ la frazione in volume della suddetta fase. Si noti che, in contrasto con Eq. (3), i tensori di localizzazione $\mathbf{A}$ e $\mathbf{B}$ non dipendono dal vettore di posizione nel volume di controllo. Tra i metodi MFAs classici si ricordano quello di Mori-Tanaka, che è l'approccio più semplice nel descrivere completamente un composito bi-fase; quello di Eshelby, che in un lavoro del 1957 studiò il campo di tensione attorno ad un'inclusione ellissoidale immersa in una matrice infinita soggetta a una deformazione uniforme (Fig. 2); il metodo di Hashin e Shtrikman.

A titolo di esempio si riportano le espressioni in forma chiusa delle proprietà elastiche di un composito bifase secondo le teorie di Eshelby, 1957 Eq. (8), Hashin-Shtrikman, 1962 Eq. (9), Mori-Tanaka, 1963 Eq. (10). Per la trattazione matematica completa si rimanda alla specifica letteratura, [2, 9-11].

$$
\begin{aligned}
& \sigma^{(i)}=E^{(i)}\left(\varepsilon_{c}-\varepsilon_{t}\right)=E^{(m)}\left(\varepsilon_{c}-\varepsilon_{\tau}\right) \\
& E_{H S}^{*}=E^{(m)}+\xi^{(r)}\left(L^{0}-E^{(m)}\right)\left[I+\xi^{(r)}\left(L^{0}+E^{(m)}\right)^{-1}\left(E^{(r)}-E^{(m)}\right)\right]^{-1} \\
& E_{M}^{*}=E^{(m)}\left\{I-\xi^{(r)}\left[\left(E^{(r)}-E^{(m)}\right)\left(S-\xi^{(r)}(S-I)\right)+E^{(m)}\right]^{-1}\left(E^{(r)}-E^{(m)}\right)\right\}^{-1}
\end{aligned}
$$

$\boldsymbol{\sigma}^{(i)}=$ tensore degli sforzi dell'inclusione; 
$\mathbf{E}^{(i)}=$ tensore di elasticità dell'inclusione;

$\mathbf{E}^{(\mathrm{m})}=$ tensore di elasticità della matrice;

$\mathbf{E}^{(\mathrm{r})}=$ tensore di elasticità di un'inclusione ellissoidica;

$\boldsymbol{\varepsilon}_{\mathrm{c}}=$ deformazione in-situ (constrained deformation) $=\mathbf{S} \boldsymbol{\varepsilon}_{\mathrm{t}}(\mathbf{S}$ è noto come il tensore di Eshelby);

$\boldsymbol{\varepsilon}_{\mathrm{t}}=$ autodeformazione (unconstrained deformation);

$\boldsymbol{\varepsilon}_{\tau}=$ autodeformazione equivalente fittizia;

$\mathbf{E}^{*}{ }_{\mathrm{HS}}, \mathbf{E}_{\mathrm{M}}^{*}=$ tensori di elasticità globali;

$\mathbf{I}=$ tensore identità;

$\xi^{(\mathrm{r})}=$ frazione in volume di inclusioni;

$\mathbf{L}^{0}=\mathbf{E}^{0}\left[\left(\mathbf{S}^{0}\right)^{-1}-\mathbf{I}\right]=$ overall constraint tensor.

I metodi variazionali o di minimo dell'energia (Hill [12], Hashin-Shtrikman [13]), sono spesso impiegati per stimare i limiti superiori e, in molti casi, inferiori dei tensori elastici, dei moduli elastici, di quelli secanti e di altre proprietà di materiali microstrutturati.

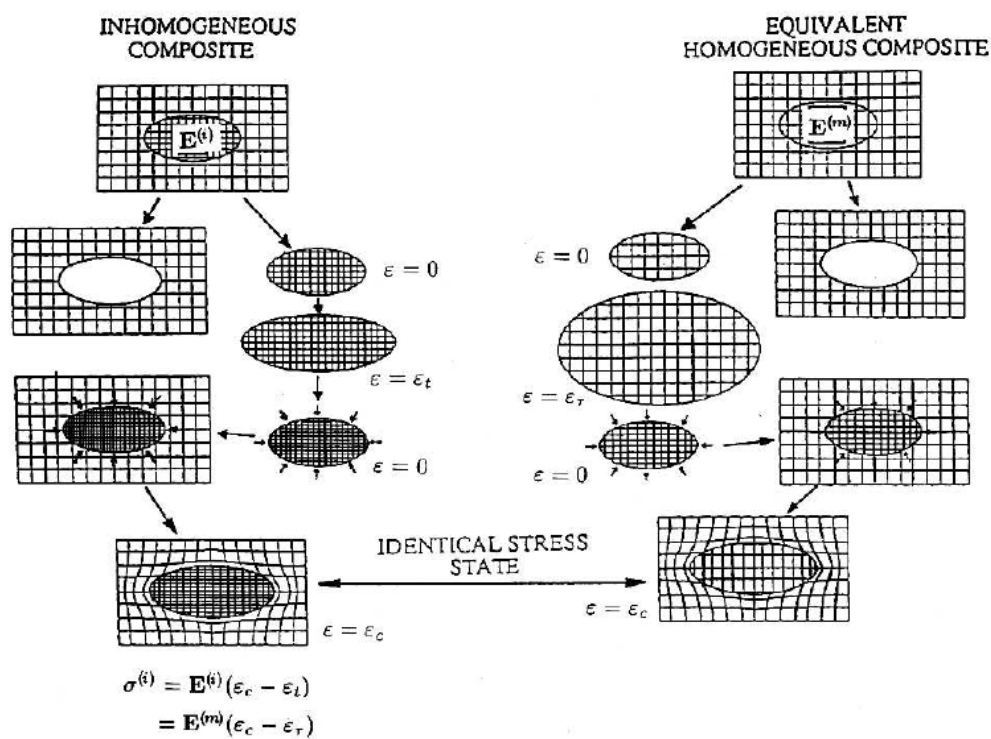

Figura 2: Schema del problema di Eshelby (1957) di determinazione del campo tensionale in un continuo elastico contenente un'inclusione soggetta alla autodeformazione $\varepsilon_{\mathrm{t}}$. Immagine tratta da [14].

\section{Metodi basati su microstrutture discrete}

Alla seconda tipologia di metodi di modellazione possono ricondursi le tecniche di studio basate su microstrutture discrete. Tra queste le più rilevanti sono la modellazione tramite microcampi periodici (celle unitarie), la modellazione a Embedded Cell, e la modellazione attraverso "finestre" campionate sulla struttura eterogenea del materiale.

Nella modellazione tramite microcampi periodici (Periodic Microfield Approaches, PMAs), che comprende i metodi a cella unitaria, la microstruttura reale è approssimata mediante un modello discreto che ripetuto uguale a se stesso riproduce una quantità comunque estesa del materiale; le fasi e il loro arrangiamento sono intrinsecamente periodici. Tali approcci, nei quali la risoluzione è solitamente affidata a metodi numerici, sono spesso utilizzati per definire dei modelli costitutivi di materiale su base microstrutturale, e per lo studio di fenomeni non-lineari, come il danneggiamento, grazie alla grande definizione che possono offrire senza impiegare grandi risorse di calcolo. Gli approcci PMAs non sono invece utilizzabili per l'analisi dell'interazione della microstruttura con fenomeni macroscopici, come ad esempio la propagazione di un difetto. La letteratura specifica dei metodi PMAs è estesissima, dato il loro ampio utilizzo; si citano qui solo alcuni riferimenti di base, [15-18].

Nell'impiego di microcampi periodici risultano particolarmente critiche le fasi di: (i) definizione del microcampo stesso, rappresentativo della struttura; (ii) definizione delle condizioni al contorno (periodicità, simmetria, antisimmetria); (iii) definizione dei carichi meccanici o termici. Tali operazioni devono essere interconnesse, come mostrato nello schema di Fig. 3, per mantenere una rappresentatività realistica della microcella. Fig. 4 chiarisce il concetto di base della modellazione a microcampi periodici, applicato alla microstruttura di una ghisa nodulare quasi interamente perlitica. Si noti come la 
microstruttura eterogenea reale, a sinistra, sia riprodotta come un array regolare di celle unitarie equispaziate, contenenti noduli di uguale dimensione e tutte le informazioni necessarie a descrivere il numero e la disposizione dei costituenti.

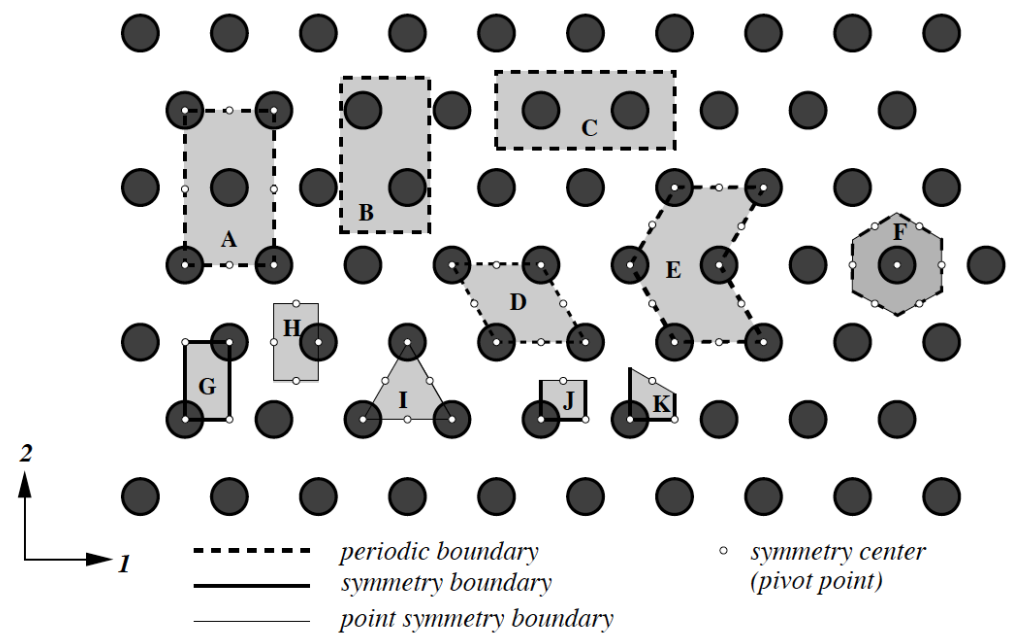

Figura 3: Schema di array esagonale periodico di inclusioni circolari (ad esempio fibre di rinforzo immerse in una matrice continua) e definizione di 11 possibili celle unitarie e delle relative condizioni al contorno [2].
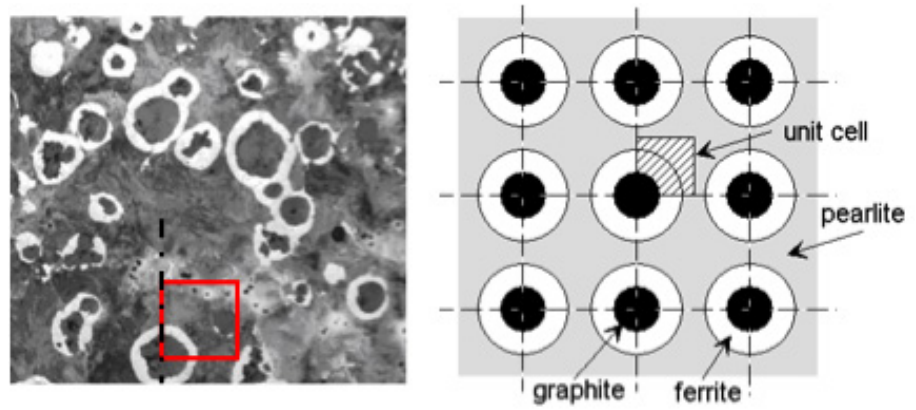

Figura 4: Schema di modellazione a cella unitaria della microstruttura di una ghisa nodulare perlitica.

Una seconda tecnica è la modellazione tramite Embedded Cell Approaches (ECAs). Secondo tale approccio, la struttura eterogenea reale è descritta da un modello di materiale costituito da un nucleo (core) contenente un arrangiamento discreto delle fasi, inserito in una regione omogenea a cui sono generalmente applicati i carichi (deformazioni e tensioni). La legge costitutiva della regione esterna al nucleo eterogeneo è solitamente quella macroscopica del materiale, nota a priori o determinabile nel caso di modelli di tipo self-consistent, [19-21].

Infine, le tecniche di modellazione che si basano sul Windowing approach, prevedono la costruzione di sub-regioni ("windows") rettangolari o esaedriche ricavate in modo casuale a partire dalla microstruttura reale, e sottoposte ad appropriate condizioni al contorno, [22]. Le finestre, che possono essere bidimensionali o tridimensionali, si possono ottenere da micrografie mediante tecniche di analisi digitale, come illustrato ad esempio in [23-25]. Questo genere di modellazione non risulta troppo adatta a descrivere la risposta globale di materiali eterogenei, quanto lo sviluppo di fenomeni locali all'interno della finestra di campionamento, come ad esempio localizzazione della deformazione in bande, sviluppo di scorrimenti, interazione tra inclusioni o particelle di rinforzo. Data la specificità dei fenomeni che si osservano in funzione della peculiare struttura esaminata, particolare attenzione va riposta nella scelta della grandezza della finestra, nel numero e nella disposizione delle inclusioni, nei tempi di calcolo. Dato che la finestra è posta casualmente al di sopra della struttura, questo è noto anche come approccio secondo degli Statistical Volume Elements (SVEs).

Uno schema riassuntivo delle tecniche appartenenti a questa tipologia di modellazione microstrutturale è illustrato nella Fig. 5. Recentemente un'interessante tecnica di modellazione windowing concettualmente nuova è stata proposta dai ricercatori del MPA (Staatliche Materialprüfungsanstalt) di Stoccarda; la tecnica prevede l'applicazione di un campo di spostamenti determinato sperimentalmente, sui bordi di un SVE numerico della microstruttura, [26]. Sperimentato su un composito $\mathrm{Al}-\mathrm{Al}_{2} \mathrm{O}_{3}$, questo approccio consente di creare un link diretto tra modellazione ed evidenza sperimentale, di importanza strategica nello studio di microcampi di deformazione e micromeccanismi di danneggiamento. 


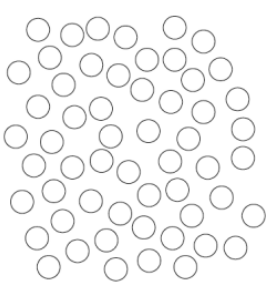

ORIGINAL CONFIGURATION

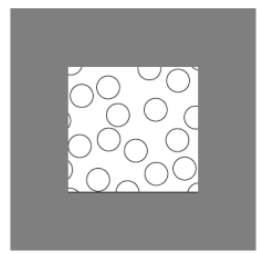

EMBEDDED CONFIGURATION
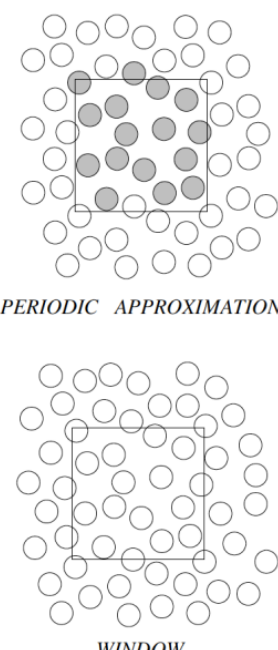

Figura 5: Tecniche di modellazione basate su microstrutture discrete: PMA, ECA e windowing approach (SVEs) [2].

\section{ALCUNE APPLICAZIONI}

\section{Modellarione tramite microcampi periodici: la cella unitaria}

$\mathrm{I}$ $\mathrm{n}$ questa sezione sono illustrate alcune applicazioni condotte dall'autore nel campo della modellazione del comportamento meccanico della ghisa nodulare, a partire dalla sua microstruttura caratteristica. È anche illustrato lo studio di recente condotto per la riproduzione della microstruttura di una lega di rame a struttura ultrafine dei grani, mediante tassellatura con l'algoritmo di Voronoi.

Come detto in precedenza, uno dei più comuni metodi di studio delle distribuzioni di sforzi e di deformazioni a livello della meso-scala è quello basato sulla riproduzione della struttura del materiale tramite una micro-geometria che si ripete periodicamente, la cella unitaria. L’uso della cella elementare nella modellazione micromeccanica si è diffuso negli ultimi anni poiché costituisce uno strumento semplice per predire la risposta costitutiva o per studiare il danneggiamento duttile di materiali disomogenei a struttura complessa, come materiali porosi o compositi a matrice metallica rinforzati con particelle o fibre. Molti lavori recenti riportano lo studio dell'evoluzione delle dimensioni di vuoti in una matrice solida; tra questi, McClintock [27] ha considerato l'evoluzione di un singolo vuoto cilindrico in una matrice infinita sottoposta a un carico assialsimmetrico; Gurson [28] ha derivato in maniera rigorosa una funzione di potenziale plastico per materiali porosi basata su una cella sferica contenente un vuoto anch'esso sferico, Tvergaard e Needleman [29, 30] hanno fatto uso della modellazione a cella elementare per studiare la localizzazione del flusso plastico ed estendere la validità del cosiddetto modello GTN alla previsione del danneggiamento e della frattura duttile. La microstruttura della ghisa sferoidale bene si presta ad una schematizzazione secondo la ripetizione di una cella elementare che contenga tutte le informazioni necessarie, poiché i noduli di grafite sono distribuiti abbastanza uniformemente nella matrice, le loro dimensioni non sono troppo dissimili, e la distribuzione delle fasi ha una caratteristica ben distinta (ciascun nodulo è incapsulato dalla fase ferritica). Diversi lavori sono reperibili in letteratura sull'utilizzo di celle unitarie per lo studio e la previsione del comportamento meccanico della ghisa nodulare, inerenti le proprietà elastiche, la simulazione del danneggiamento, o l'effetto della presenza di tensioni termiche, [31-34]. In un lavoro del presente autore [35] si è presentato un modello a cella unitaria della ghisa nodulare, mostrato in Fig. 6, che comprendesse anche la fase perlitica nella matrice ferrosa. Lo studio è stato incentrato sulla risposta meccanica della microstruttura al variare dei parametri caratteristici come il contenuto e la disposizione delle fasi.

Le leggi costitutive delle fasi presenti nel materiale (ferrite, perlite, grafite) sono desunte da dati di letteratura. I risultati della modellazione sono presentati in termini di risposta alla trazione monoassiale della cella, una volta adottata una procedura di omogeneizzazione basata sul volume di riferimento che consenta di rilevare il comportamento del materiale a livello macroscopico.

I diagrammi di Fig. 7 riportano alcuni dei risultati ottenibili con l'approccio a cella unitaria. Per quanto riguarda le proprietà elastiche, il confronto con risultati sperimentali e con tecniche di modellazione Mori-Tanaka e HashinShtrikman, evidenzia come l'approccio a cella unitaria sia delimitato inferiormente e superiormente dai due metodi mean field, e come d'altra parte i valori sperimentali del modulo elastico effettivo siano dispersi attorno ai risultati delle 
modellazioni a causa di altri fattori microstrutturali (concentrazione delle tensioni, micro-plasticizzazioni locali, discontinuità). La predizione della resistenza - Fig. 7(b) - evidenzia un maggior accordo con i dati sperimentali, indicando una notevole capacità della cella unitaria di riprodurre i fenomeni plastici in microstrutture abbastanza regolari e ripetitive come quella della ghisa nodulare.

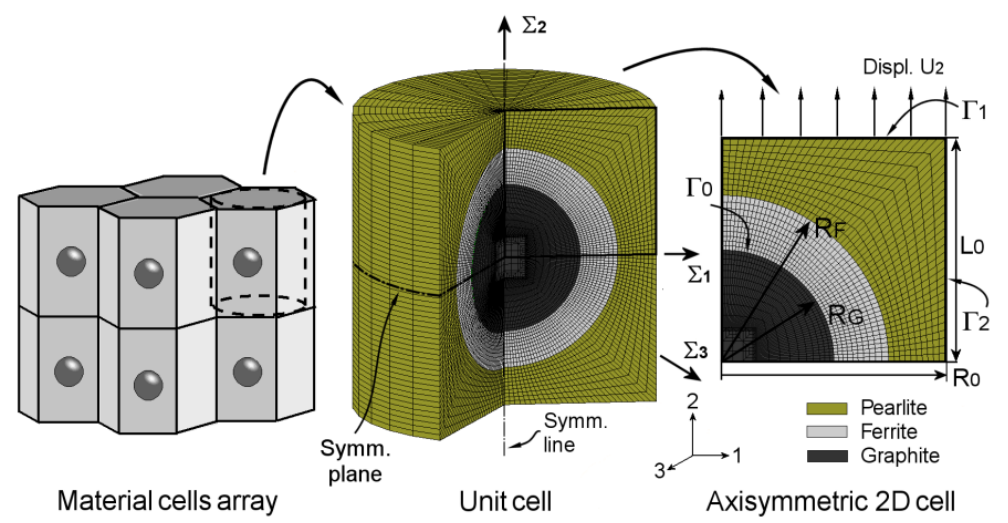

Figura 6: Modellazione della ghisa nodulare mediante cella assialsimmetrica, [35].

Altre modellazioni successive a quella descritta in Fig. 6 sono state condotte con celle unitarie più raffinate, finalizzate da una parte allo studio comparativo dell'effetto di costrizione della deformazione introdotta dai modelli di tipo assialsimmetrico, e dall'altra all'analisi più dettagliata della microstruttura. Con tali modelli, ad esempio, è stata indagata la risposta meccanica di una microstruttura con due diverse dimensioni caratteristiche dei noduli, individuate mediante considerazioni statistiche su micrografie reali, e variando la loro disposizione spaziale reciproca, [37].

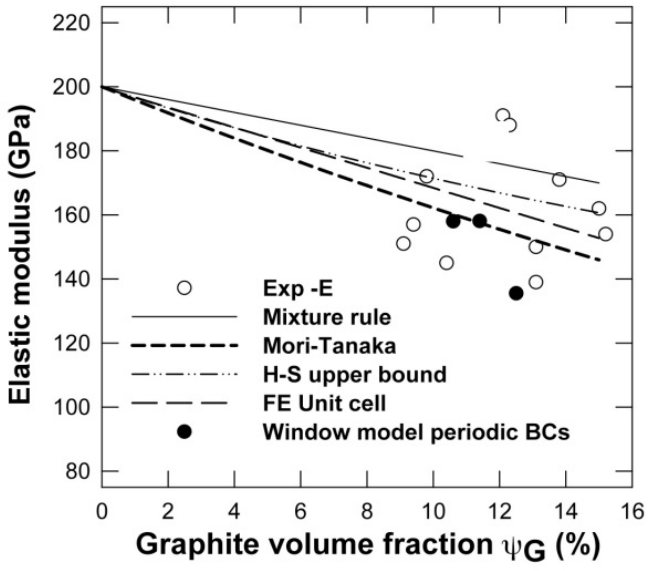

(a)

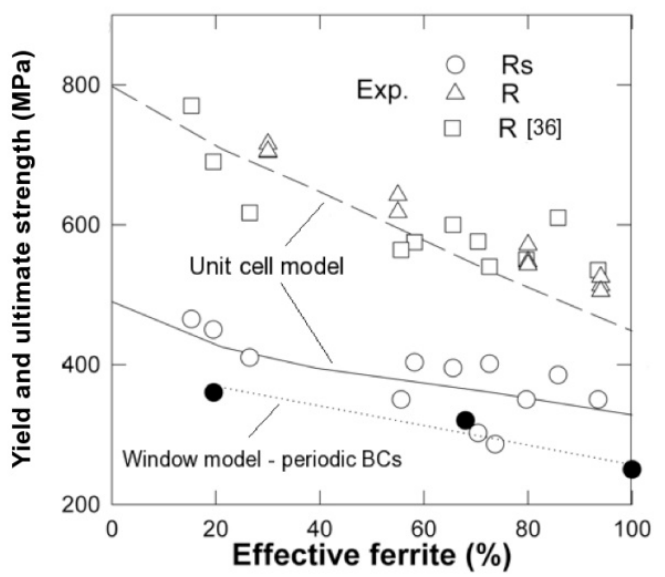

(b)

Figura 7: (a) effetto del contenuto in grafite sulle proprietà elastiche della ghisa nodulare;

(b) effetto del contenuto in ferrite sulla resistenza della ghisa nodulare [37].

Alcune celle unitarie di questo tipo sono illustrate in Fig. 8: qui si hanno celle tridimensionali con percentuale dei costituenti variabile, e disposizione reciproca dei noduli di grafite (riprodotti come cavità sferiche) ad array incrociato o sfalsato. La cella unitaria offre di fatto la possibilità di studiare, impiegando modeste risorse di modellazione e di calcolo, diverse configurazioni (periodiche) delle inclusioni nella matrice, analizzandone gli effetti sulla rigidezza del materiale, sul flusso plastico delle tensioni, e in generale sulla risposta effettiva del materiale.

Lo studio comparativo di questi diversi microcampi periodici ha evidenziato una certa sensibilità della cella unitaria nel descrivere la risposta meccanica del materiale, come mostrato dai diagrammi di Fig. 9. Nel confronto, illustrato in Fig. 9(a), gli effetti della modellazione tridimensionale su due microstrutture a diverso contenuto della fase ferritica mostrano una sostanziale equivalenza nel descrivere il comportamento elastico, mentre viene riprodotto un incrudimento plastico maggiore nel caso della cella assialsimmetrica. 


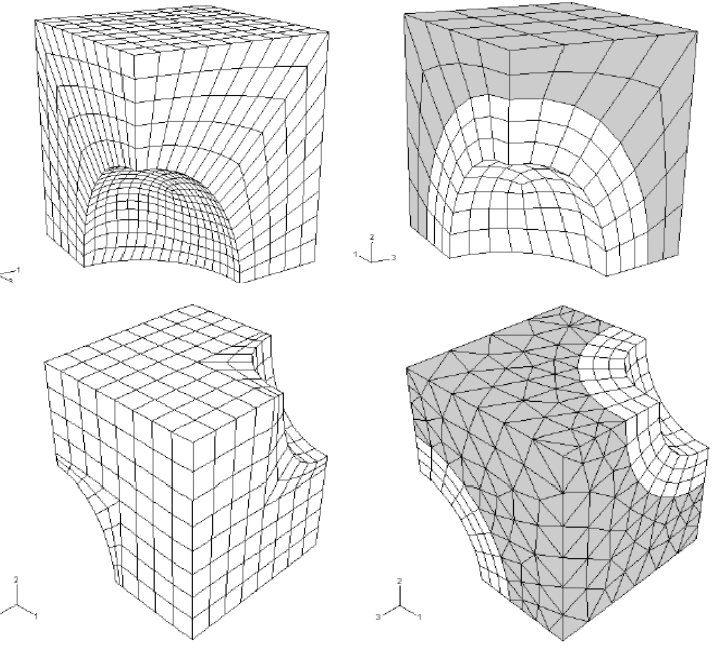

Figura 8: Microcelle unitarie ad una e due cavità per la modellazione della ghisa nodulare.

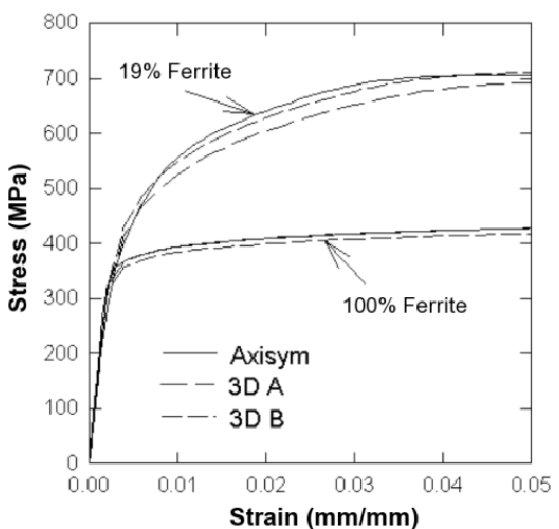

(a)

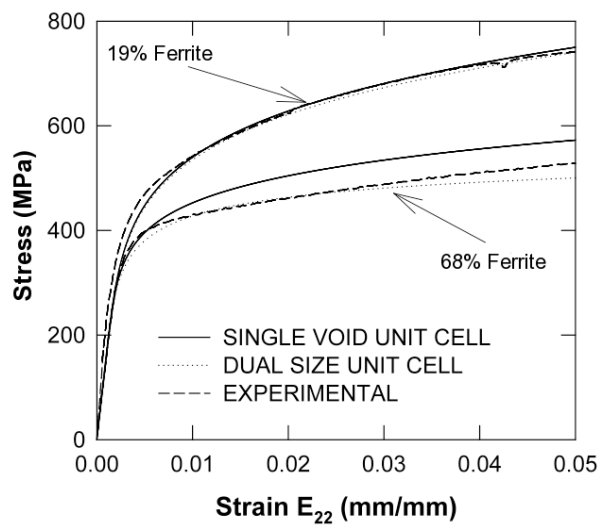

(b)

Figura 9: (a) risposta della ghisa nodulare simulata con modelli di cella assialsimmetrica e tridimensionale (modelli 3D A e 3D B); (b) effetto della modellazione a cella assialsimmetrica con doppia cavità [37].

D'altra parte, l'introduzione di una seconda cavità nella cella al fine di valutare l'interazione reciproca di due vuoti con diverso volume, mostra come, a parità del contenuto in grafite, un nodulo piccolo assieme ad un nodulo grande sia una condizione peggiorativa nella risposta plastica rispetto alla presenza di noduli di grandezza uniforme. Dal punto di vista sperimentale, si veda Fig. 9(b), si deduce che la tipologia di cella assialsimmetrica "più adatta" dipende dalla quantità e disposizione dei costituenti: la cella con singola cavità è un valido strumento interpretativo nel caso di configurazione bull's eye della struttura (limitata presenza della fase soft ferritica attorno ai noduli), mentre la modellazione mediante celle con doppia cavità trova un maggiore riscontro nel descrivere il comportamento della ghisa nodulare quando viene incrementata la presenza della fase più dura e resistente nella matrice.

\section{Modellazione di microstrutture discrete: Embedded Cell e Windowing Apporaches}

In questa sezione sono presentati alcuni esempi di modellazione microstrutturale di tipo Embedded e Windowing applicati alla ghisa nodulare a matrice mista ferritico/perlitica. Questi approcci di modellazione si basano sulla riproduzione di microstrutture discrete, generalmente a partire da micrografie o rilevamenti microstrutturali compiuti su sezioni metallografiche. Rispetto alla modellazione con microvolumi periodici, i modelli windowing sono impiegati per lo studio di fenomeni locali sui quali hanno grande influenza le caratteristiche morfologiche specifiche della struttura eterogenea. Un modello window può essere impiegato ad esempio per valutare la distribuzione delle tensioni a livello microstrutturale responsabili dei meccanismi di danneggiamento tipici della ghisa nodulare, come quelli illustrati in Fig. 10.

La creazione, la discretizzazione in elementi finiti, e la risoluzione numerica, sono per questi modelli alquanto più laboriose rispetto ai modelli a cella unitaria. In Fig. 11 è presentato un modello Embedded di una ghisa nodulare con il $9 \%$ di contenuto in grafite, il $63 \%$ di perlite e il $37 \%$ di ferrite, e di caratteristiche meccaniche note. Il modello, che consta in 
circa 50 mila elementi plane strain, viene costruito a partire da una specifica micrografia, inserendo la finestra (embedding) che contiene le informazioni sulla struttura eterogenea in un continuo omogeneo di dimensioni opportune, e la cui legge costitutiva è desunta dalla risposta macroscopica. Le leggi costitutive delle fasi microstrutturali, qui nella forma di modelli di Ramberg-Osgood, sono invece tratte dalla letteratura. Questo genere di modellazione offre il doppio vantaggio di imporre automaticamente appropriate condizioni al contorno alla finestra di analisi, e di ottenere una risposta effettiva già omogeneizzata in quanto al perimetro possono essere applicati direttamente degli spostamenti noti.

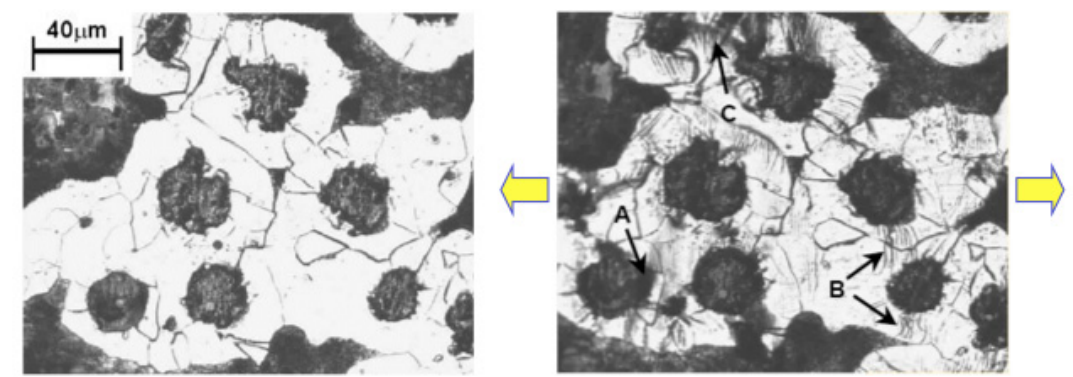

Figura 10: Meccanismi di danneggiamento in una ghisa nodulare ferritico/perlitica; $\mathrm{A}=$ debonding dei noduli; $\mathrm{B}=$ localizzazioni di deformazione plastica; $\mathrm{C}=$ bridging di microcricche, [38].

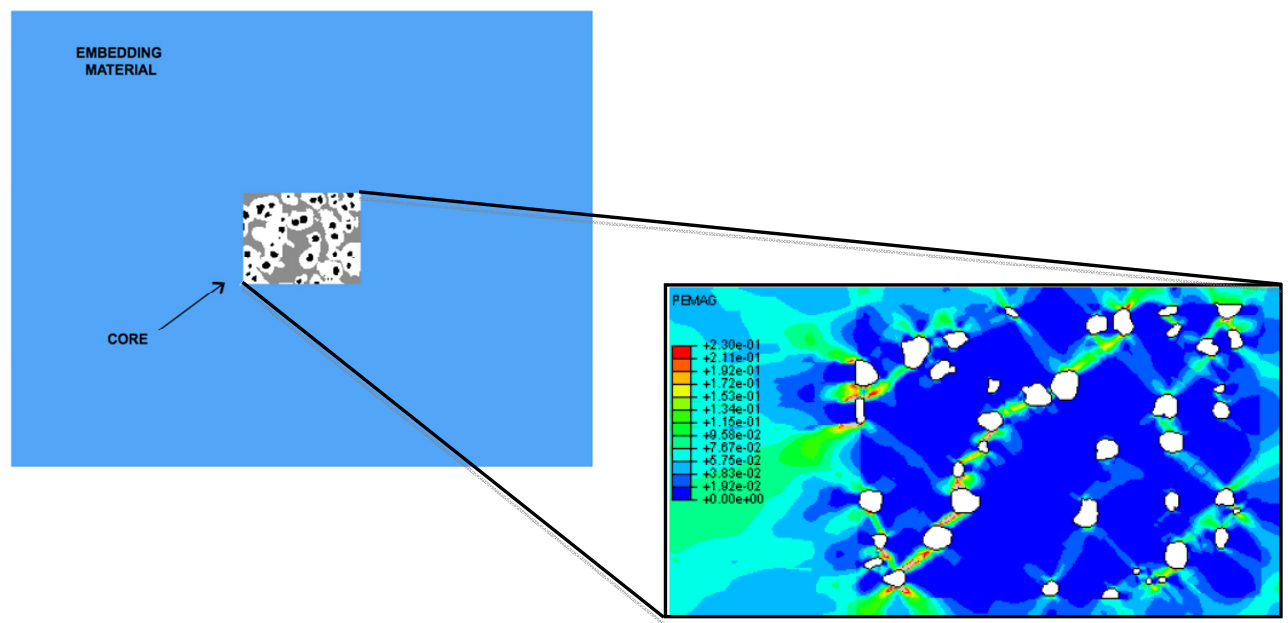

Figura 11: Modello tipo Embedded cell, e distribuzione della risultante della deformazione plastica in seguito all'applicazione di un carico in direzione orizzontale.

I risultati ottenibili sono generalmente finalizzati allo studio dell'andamento del carico nella microstruttura e della distribuzione della deformazione, nonché dell'interazione reciproca tra i costituenti valutata nella configurazione reale. Il contour mostrato a titolo di esempio nella Fig. 11, riporta la distribuzione della risultante della componente plastica di deformazione nella microstruttura. Esso mostra come la comparsa di fenomeni di localizzazione generino deformazioni ben maggiori di quelle effettive, e come l'interazione tra noduli opportunamente orientati siano responsabili della nascita di bande di scorrimento. Fenomeni di danneggiamento microscopico come quelli illustrati nella micrografia di Fig. 10, possono trovare con questo modello un'interpretazione quantitativa e stimolare l'ottimizzazione della microstruttura e quindi delle prestazioni del materiale.

Una valida alternativa alla modellazione di tipo Embedded è, come visto nella sezione Approcci e metodi della modellazione micromeccanica, la modellazione di tipo windowing. A parità di microstruttura in esame, questa differisce dalla precedente sostanzialmente per l'estensione geometrica del modello, che ne favorisce la rapidità di risoluzione, e per le condizioni al contorno impostate.

In Fig. 12 sono mostrati due modelli di questo tipo della stessa ghisa nodulare del modello Embedded, che differiscono tra loro per la tipologia di vincolo e di carico (spostamenti $\mathrm{u}_{1}$ e $\mathrm{u}_{2}$ applicati uniformemente sui lati $\Gamma_{\mathrm{L}}$ ). Una questione delicata che sorge quando si adotta questo approccio, è la scelta della dimensione ottimale della finestra di "campionamento"; se da una parte un modello di grande estensione riesce a catturare maggiori informazioni ed è statisticamente più 
rappresentativo del materiale, dall'altra le risorse e i tempi computazionali richiesti per la sua risoluzione possono diventare proibitivi.

Le curve in Fig. 13(a) mostrano che la risposta meccanica del modello di Fig. 12, pressoché analoga lungo le due direzioni di caricamento, differisce sostanzialmente sia nel campo elastico che in quello plastico dalla risposta di un modello windowing delle dimensioni pari a un quarto (small window model). Uno studio di questo tipo potrebbe essere utile per determinare la grandezza ideale della finestra di riproduzione.

A differenza dell'impiego di micro campi periodici, questi ultimi due tipi di simulazione, comunque, non si prestano in maniera rigorosa a riprodurre la risposta effettiva di una certa microstruttura. Uno dei punti deboli delle tecniche Embedded e Windowing, infatti, è la loro eccessiva cedevolezza a causa dell'assoluta mancanza di costrizione della deformazione; inoltre, con questi modelli è perduta la condizione di periodicità della struttura. Uno sforzo in tale direzione è però possibile impostando delle particolari condizioni al contorno di periodicità sul perimetro del modello (periodic window), [39].
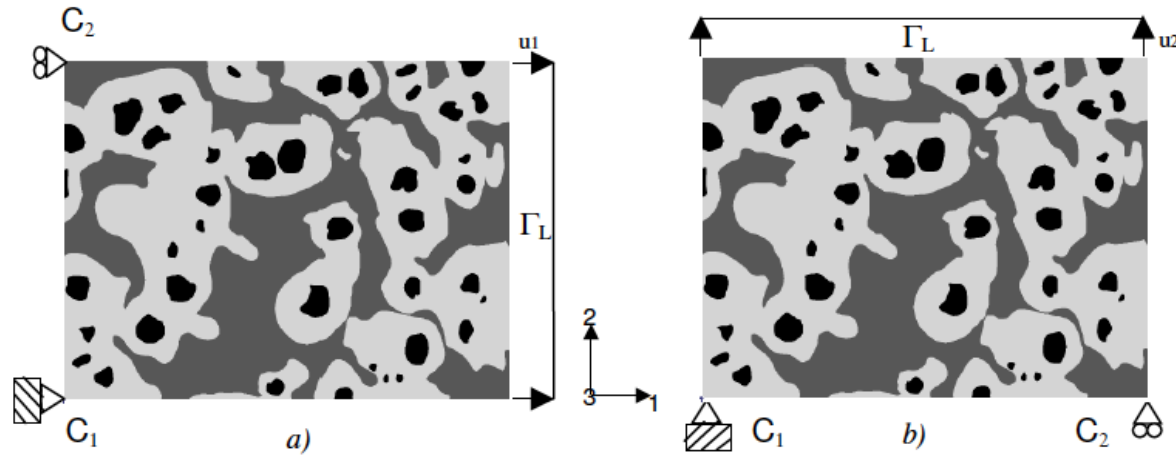

Figura 12: Modello windowing della ghisa nodulare.

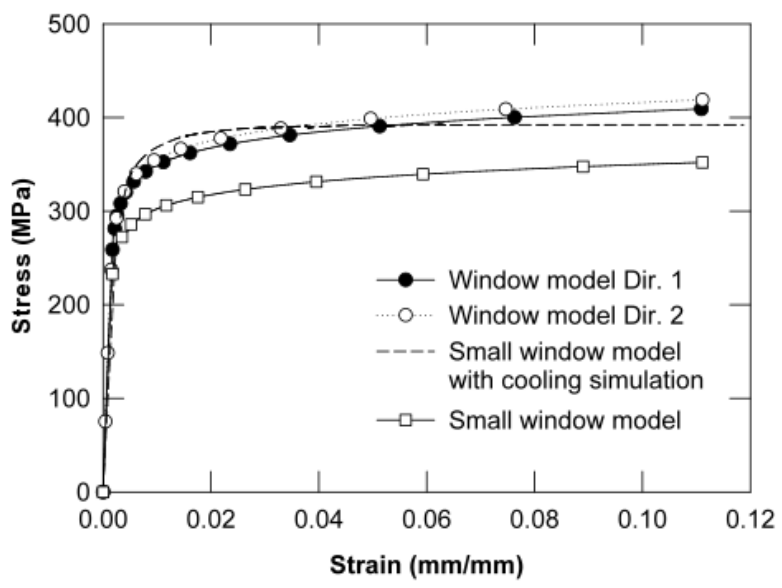

(a)

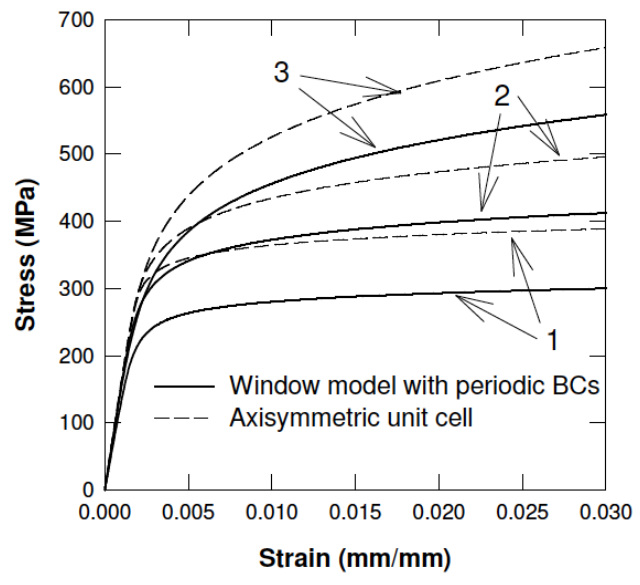

(b)

Figura 13: Risposte meccaniche della ghisa nodulare simulate con modelli discreti della microstruttura:

(a) curve di trazione dei modelli di Fig. 12; (b) risposte meccaniche dei modelli a condizioni al contorno periodiche di Fig. $14(1=100 \% \mathrm{Fe} ; 2=68 \% \mathrm{Fe} ; 3=19 \% \mathrm{Fe})$ [37].

Con riferimento allo schema di Fig. 14, che mostra un altro modello window di una ghisa nodulare a struttura interamente ferrica (GJS400), le condizioni al contorno di periodicità sono esprimibili mediante le Eq. (11a-f):

$$
\begin{aligned}
& \left.\overline{\boldsymbol{u}}\right|_{\Gamma_{34}}-\left.\overline{\boldsymbol{u}}\right|_{C_{4}}=\left.\overline{\boldsymbol{u}}\right|_{\Gamma_{12}}-\left.\overline{\boldsymbol{u}}\right|_{C_{1}} \\
& \left.\overline{\boldsymbol{u}}\right|_{\Gamma_{14}}-\left.\overline{\boldsymbol{u}}\right|_{C_{1}}=\left.\overline{\boldsymbol{u}}\right|_{\Gamma_{23}}-\left.\overline{\boldsymbol{u}}\right|_{C_{2}} \\
& \left.\overline{\bar{\sigma}} \cdot \overline{\boldsymbol{n}}\right|_{\Gamma_{12}}=-\left.\bar{\sigma} \cdot \overline{\boldsymbol{n}}\right|_{\Gamma_{34}}
\end{aligned}
$$

$$
\begin{aligned}
& \left.\bar{\sigma} \cdot \overline{\boldsymbol{n}}\right|_{\Gamma_{14}}=-\left.\bar{\sigma} \cdot \overline{\boldsymbol{n}}\right|_{\Gamma_{23}} \\
& \left.\boldsymbol{u}_{2}\right|_{C_{1}}=\left.\boldsymbol{u}_{2}\right|_{C_{2}} \\
& \left.\overline{\boldsymbol{u}}\right|_{C_{3}}-\left.\overline{\boldsymbol{u}}\right|_{C_{2}}=\left.\overline{\boldsymbol{u}}\right|_{C_{4}}-\left.\overline{\boldsymbol{u}}\right|_{C_{1}}
\end{aligned}
$$



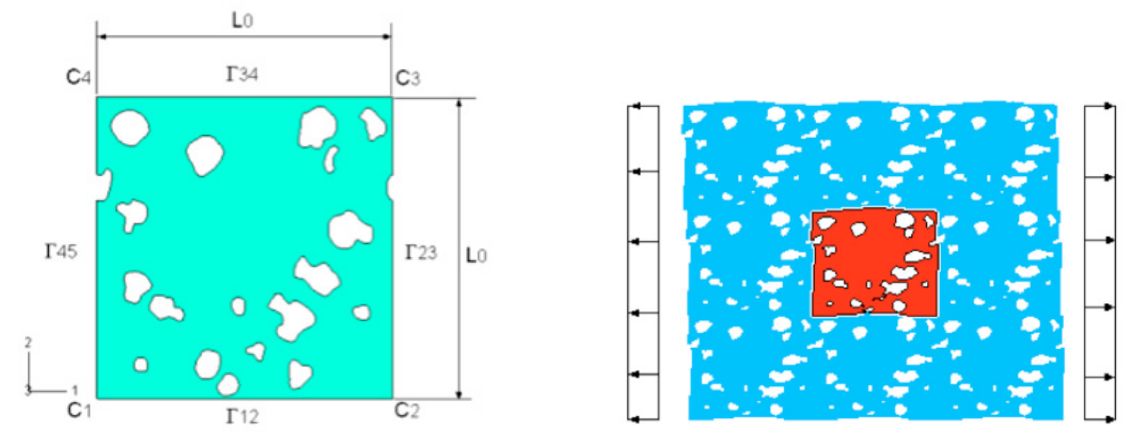

Figura 14: Un modello window con condizioni al contorno opportune può riprodurre un microcampo periodico.

con la seguente notazione:

$\bar{u}=\left(u_{1}, u_{2}\right)=$ vettore spostamento;

$-$

$n=$ vettore normale;

$\mathrm{C}_{1,2,3,4}=$ boundaries del modello;

$\sigma=$ vettore delle tensioni risultanti.

Nello specifico, le relazioni scritte impongono la simmetria degli spostamenti nodali sui lati affacciati - Eq. (11a) e (11b) l'equilibrio delle forze che si generano sugli stessi lati - Eq. (11c) e (11d) - e vietano la traslazione - Eq. (11e) - e la rotazione - Eq. (11f) - della finestra. La deformazione nel modello può allora essere originata applicando uno spostamento lungo una direzione a piacere al nodo in $\mathrm{C}_{3}$, che non possiede gradi di libertà costretti. Altre informazioni maggiormente specifiche su questa tecnica di modellazione sono reperibili ad esempio in [40].
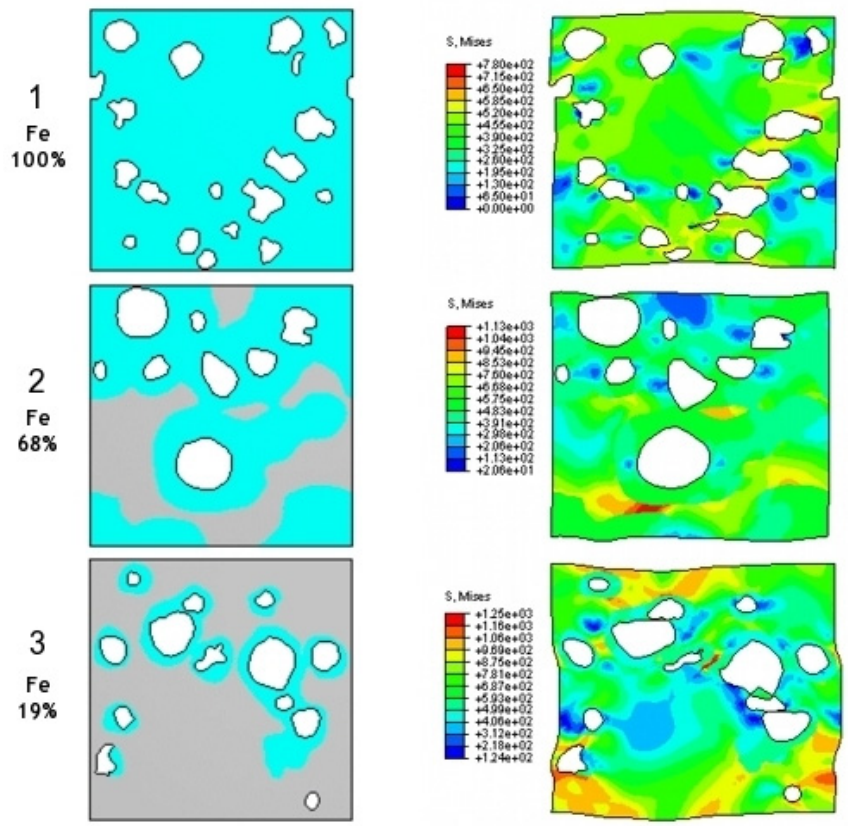

Figura 15: distribuzione dello sforzo in tre microstrutture eterogenea di ghisa nodulare simulate con modelli windowing a condizioni al contorno periodiche (Eq. 11).

Gli aspetti che questo tipo di modellazione mette in luce non differiscono significativamente da quelli già evidenziati dai modelli window appena discussi. In Fig 15 sono illustrate le distribuzioni dello sforzo equivalente in tre microstrutture di ghisa nodulare, variabili per percentuali dei costituenti nella matrice, sottoposte ad una deformazione effettiva del 3\%. Le distribuzioni bene evidenziano la disuniformità degli sforzi, corrispondenti allo stesso livello di deformazione mesoscopica imposta. Lo stato locale di sforzo è affetto dalla presenza di cavità, dalla conformazione della microstruttura e dalle 
discontinuità tra le fasi. La deformazione nella ghisa a matrice interamente ferritica si localizza lungo bande a $45^{\circ}$ che rappresentano "punti deboli"; nella microstruttura mista la tensione si localizza in una striscia perlitica che unisce due subregioni ferritiche, mentre nella microstruttura quasi completamente perlitica si presenta un'alta disomogeneità dello sforzo, sebbene la ferrite presente attorno ai noduli sia sottoposta ad uno sforzo pressoché costante. Quest'ultimo è di entità ridotta poiché la libera deformazione del materiale è impedita dalle zone perlitiche circostanti. Il modello interpreta quindi correttamente $\mathrm{i}$ meccanismi di cedimento fragile osservati sperimentalmente anche nella fase ferritica in ghise sferoidali a matrice quasi interamente perlitica, [41, 42].

Analizzando la risposta di resistenza a trazione di questi tre modelli, si osserva, a parità delle percentuali di costituenti nella microstruttura, un comportamento sostanzialmente più cedevole rispetto alla cella assialsimmetrica, si vedano le curve di Fig 13(b), in particolar modo quando si incrementa la percentuale di ferrite. Questo aspetto conferma l'influenza della disomogeneità e della localizzazione della deformazione associate alle discontinuità microstrutturali che con tale tecnica si riescono a riprodurre.

\section{Generazione di microstrutture statisticamente equivalenti}

Un ultimo cenno vuole essere rivolto al concetto legato alla generazione di microstrutture statisticamente equivalenti di materiali a struttura in qualche modo eterogenea. Due strutture si definiscono statisticamente equivalenti quando possiedono uguali valori dei loro descrittori statistici.
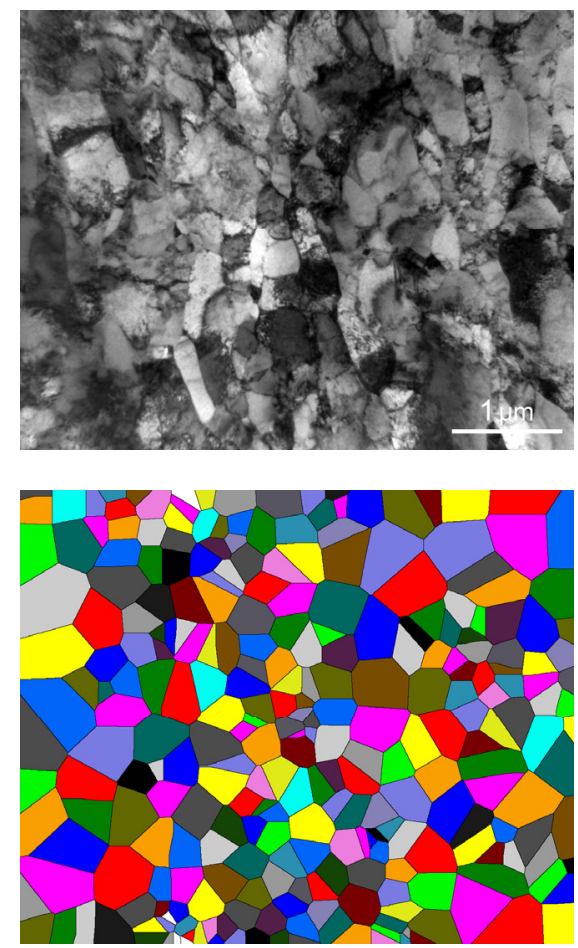

Figura 16: Microfrafia TEM di una lega di rame a struttura ultrafine del grano e tassellatura Voronoi statisticamente equivalente.

Una delle tecniche maggiormente utilizzate per generare modelli geometrici equivalenti è la tassellatura o decomposizione Voronoi (dal nome del matematico Georgy Voronoi risalente ai primi del '900), originariamente applicata in geofisica e meteorologia. L'algoritmo di Voronoi consiste nel definire un certo numero di regioni (celle), a partire da un insieme discreto di punti, con la condizione che i lati di ciascuna cella siano equidistanti da tali punti. Una tassellatura di Voronoi può essere definita con alcune specifiche proprietà, come l'unicità di ciascuna cella, i limiti inferiore e superiore e il valore medio delle dimensioni delle celle, il numero minimo e massimo di lati, i valori estremi degli angoli. La tassellatura Voronoi è producibile nel piano o anche nello spazio. La formulazione matematica completa dell'algoritmo di Voronoi può essere reperita ad esempio in [43].

Le celle di un reticolo così creato sono statisticamente rappresentative, nelle due o tre dimensioni, della struttura a grani di un materiale metallico policristallino. L’equivalenza statistica della tassellatura nei confronti di una struttura reale, può imporsi nella dimensione media dei grani (misurata ad esempio tramite l'area, il lato maggiore, il prodotto lato maggiore 
per lato minore o l'area del cerchio equivalente), nella loro orientazione, o in altri fattori geometrici. Il presente autore è al momento impegnato nella modellazione dell'effetto della dimensione media del grano sulla resistenza alla propagazione di difetti di fatica nel rame. In particolare, con riferimento ad una campagna di prove sperimentali da poco terminate, è in esame la lega di rame a struttura ultrafine mostrata in Fig. 16. In questo specifico caso, la generazione delle celle di Voronoi è stata effettuata determinando i nodi interni a ciascuna cella a partire dal riconoscimento automatico del gradiente di grigio in una micrografia TEM del tutto analoga a quella di Fig. 16. Ciascun punto viene determinato come un punto di massimo del gradiente di colore in una determinata area, e l'equivalenza statistica della distribuzione della dimensione dei grani, verificata dopo ogni passaggio, è ottenuta tramite la continua modifica del livello di soglia del riconoscimento dei toni di grigio. Il lavoro è stato condotto con il programma di analisi digitale "ImageJ" scaricabile gratuitamente dal sito http://rsbweb.nih.gov/ij.

\section{CONSIDERAZIONI CONCLUSIVE}

I n questo lavoro si sono presentate alcune tecniche di modellazione microstrutturale utilizzate per lo studio della meccanica dei materiali eterogenei. Assieme ad alcuni cenni al concetto di "scala di indagine" e alle teorie classiche e di base della Meso- e Micromeccanica, si sono illustrati i risultati di un certo numero di casi di studio concreti. Nella discussione critica degli aspetti più interessanti delle analisi mostrate, si è cercato di evidenziare punti di forza e possibilità di ricerca offerte, ma anche vincoli e limitazioni di ciascun metodo. L'approccio micromeccanico applicato a un materiale a struttura eterogenea come la ghisa nodulare, si dimostra in definitiva uno strumento indispensabile sia per l'analisi e l'interpretazione di fenomeni locali che dipendono dalla microstruttura, sia per la previsione del suo comportamento elastico e plastico.

\section{BIBLIOGRAFIA}

[1] S. Nemat-Nasser, M. Hori, Micromechanics: overall properties of heterogeneous materials. North-Holland, Amsterdam (1993).

[2] H. J. Böhm, Mechanics of Microstructured Materials. CISM Courses and Lectures, Springer-Verlag, Vienna, 464 (2004).

[3] L. L. Mishnaevsky, S. Schmauder, Applied Mechanics Review, 54(1) (2001) 49.

[4] S. Torquato, Theory of composite materials. Oxford University Press, London (2001).

[5] P.V. Makarov, S. Schmauder, O.I. Cherepanov, I.Yu. Smolin, V.A. Romanova, R.R. Balokhonov,D.Yu. Saraev, E. Soppa, P. Kizler, G. Fischer, S. Hu, M. Ludwig, Theoretical and Applied Fracture Mechanics, 37 (2001) 183-.

[6] J. Aboudi, Mechanics of composite materials. Elsevier, Amsterdam (1991).

[7] R. Hill, J. Mech. Phys. Solids, 15 (1965) 89.

[8] Z. Hashin, S. Shtrikman, J. Mech. Phys. Sol., 11 (1963) 127.

[9] T. Mori, K. Tanaka, Acta Metall., 21 (1973) 571.

[10] J. D. Eshelby, In: Proc. R. Soc. London, A 241 (1957) 376.

[11] H. J. Böhm, A short introduction to basic aspects of continuum micromechanics. CDL-FMD Report, 3 (1998).

[12] R. Hill, In: Proc. Phys. Soc., A65 (1952) 349.

[13] Z. Hashin, S. Shtrikman, J. Mech. Phys. Solids, 10 (1962) 335.

[14] P. J. Withers, Philosophical Magazine A, 59(4) (1989) 759.

[15] Y. Bansal, M.-J. Pindera, Int. J. Plasticity, 22 (2006) 775.

[16] S. Li, A. Wongsto, Mechanics of Materials, 36 (2004) 543.

[17] M.-J. Pindera, H. Khatama, A.S. Dragob, Y. Bansal, Composites: Part B, 40 (2009) 349.

[18] I. Saiki, K. Teradab, K. Ikedab, M. Hori, Comput. Methods Appl. Mech. Engrg., 191 (2002) 2561.

[19] N. Takano, M. Zako, T. Okazaki, JSME Int. J. Srs., A44 (2001) 602.

[20] G. L. Heness, B. Ben-Nissan, L. H. Gan, Y. W. Mai, Comput. Mater. Sci., 13 (1999) 259.

[21] H. R. Chen, Q. S. Yang, F. W. Williams, Comput. Mater. Sci., 2 (1994) 301.

[22] M. Ostoja-Starzewski, Int. J. Sol. Struct., 35 (1998) 2429.

[23] Z. Shan, M. Arun, A. M. Gokhale, Int. J. Plasticity, 20 (2004) 1347.

[24] Z. Shan, A. M. Gokhale, Acta Mater., (2001) 2001.

[25] N. Takano, M. Zako, F. Kubo, K. Kimura, Int. J. Sol. Struct., 40 (2003) 1225. 
[26] E. Soppa, S. Schmauder, G. Fischer, J. Brollo, U. Weber, Comput. Mater. Sci., 28 (2003) 574.

[27] F. A. McClintock, J. Appl. Mech., 35 (1968) 363.

[28] A. L. Gurson, J. Engng. Mater. Technol., 99 (1977) 2.

[29] V. Tvergaard, J. Mech. Phys. Solids, 30 (1982) 265.

[30] A. Needleman, V. Tvergaard, Acta Metall., 32 (1984) 157.

[31] W. Brocks, S. Hao, D. Steglich, J. de Physique IV, 6 C6 (1996) 43.

[32] M. Kuna, D.-Z. Sun, J. de Physique IV, 6 C6 (1996) 113.

[33] N. Bonora, A. Ruggiero, Int. J. Sol. Struct., 42 (2005) 1401.

[34] D. Steglich, W. Brocks, Comput. Mater. Science, 9 (1997) 7.

[35] L. Collini, G. Nicoletto, J. Strain Analysis, 40 (2005) 107.

[36] W. Brocks, G. Pusch, W. Baer, D. Steglich, Anwendung mikromechanischer Modelle der Werkstoffschädigung zur gefügeabhängigen Bewertung der Zähigkeit von duktilen Gußeisenwerkstoffen, Final report of DFG-Project Br521/6 and Pu104/4, Geesthacht and Freiberg, Germany (1996).

[37] L.Collini, Micromechanical modeling of the elasto-plastic behavior of heterogeneous nodular cast iron. Tesi di dottorato in Ingegneria Industriale, XVII ciclo (2005).

[38] L. Collini, P. Bujnova, In: Procs. of $3^{\text {rd }}$ YSESM, Porretta Terme, Italy (2004) 61.

[39] L. Collini, G. Nicoletto, In: Atti XXXIV Convegno AIAS, Milano (2005).

[40] R. J. Smit, W. A. Brekelmans, H. E. Meijer, Comput. Methods Appl. Mech. Engrg., 155 (1998) 181.

[41] G. Nicoletto, R. Konečna, B. Hadzimova, L. Collini, In: Atti XXXI Convegno AIAS, Parma (2002).

[42] F. Iacoviello, V. Di Cocco, F. Franzese, Workshop IGF, Forni di Sopra (2010).

[43] V. Du, Q. Faber, M. Gunzburger, SIAM Review, 41(4) (1999) 637. 